
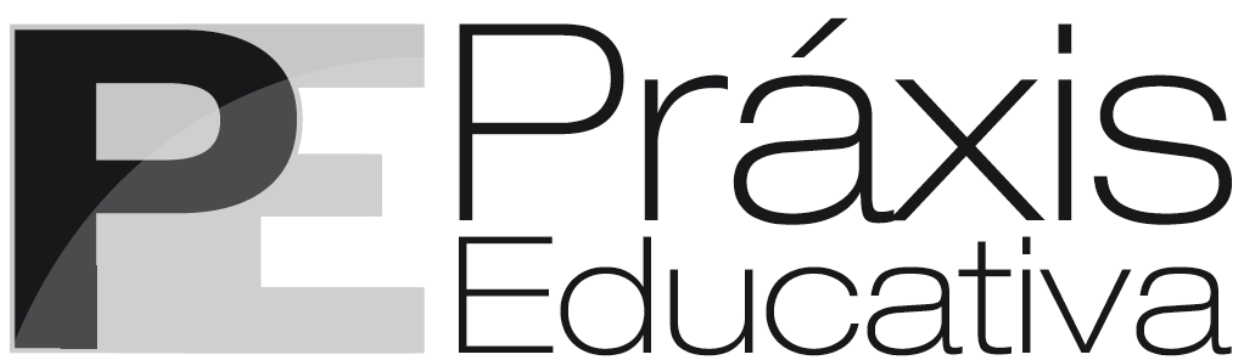

ISSN 1809-4031

eISSN 1809-4309

https://doi.org/10.5212/PraxEduc.v.16.16583.041

\title{
La recuperación de la praxis en el desarrollo curricular de la formación docente. El caso de la provincia de Buenos Aires*
}

\section{A recuperação da práxis no desenvolvimento curricular da formação de professores: o caso da província de Buenos Aires}

\section{The recovery of praxis in the development of teacher training curricula: the case of the province of Buenos Aires}

\author{
Noralí Boulan ${ }^{* *}$ \\ (iD) https://orcid.org/0000-0002-4600-0976
}

Resumen: El presente artículo es producto de la investigación doctoral desarrollada entre 2015 y 2019 en la que se analizó el proceso de reformulación curricular para la formación de profesores del Nivel Primario en la Provincia de Buenos Aires, así como también, orienta la hipótesis que dicho proceso implicó, una reestructuración de los sentidos de la enseñanza a partir de la centralidad de la categoría "praxis" en la formación profesional. Asimismo, el análisis de las fuentes primarias, bibliografía especializada y las entrevistas realizadas a los sujetos involucrados permiten afirmar que el curriculum es comprendido como práctica discursiva que procura configurar identidades, aunque es posible observar en las resistencias que los docentes establecen al respecto, durante el proceso de desarrollo curricular en dicha jurisdicción entre los años 2004 y 2007, un proceso de negociación permanente entre la interpelación oficial y los sujetos destinatarios de dicha política.

Palabras clave: Formación docente. Curriculum. Praxis.

\footnotetext{
* Este artículo es producto de la Tesis Doctoral "Políticas de formación de profesores de nivel primario en la provincia de Buenos Aires. Discursos, curriculum y procesos identificatorios (2006-2016)". Doctorado en Ciencias de la Educación. Universidad Nacional de La Plata (UNLP), Argentina. La misma fue financiada mediante una Beca de Finalización de Doctorado por el Consejo Nacional de Investigaciones Científicas y Técnicas (CONICET), Argentina.

** Doctora en Ciencias de la Educación por la Universidad Nacional de La Plata, Argentina. Becaria Posdoctoral del Consejo Nacional de Investigaciones Científicas y Técnicas (CONICET). Participa de Proyectos de Investigación y Desarrollo en el Instituto de Investigaciones en Humanidades y Ciencias Sociales de la Facultad de Humanidades y Ciencias de la Educación (IdIHCS-FaHCE) de la Universidad Nacional de La Plata. Actualmente se desempeña como Tutora del Programa de Doctorado en Estudios Latinoamericanos de la Universidad Nacional de La Plata (UNLP), y como docente en Institutos Superiores de Formación Docente (ISFD) de la Provincia de Buenos Aires (DGCyE). Se especializa en temáticas relacionadas a políticas curriculares y educativas vinculadas a la formación docente. E-mail: $<$ noraliboulan@gmail.com>.
} 
La recuperación de la praxis en el desarrollo curricular de la formación docente. El caso de la província...

Resumo: Este artigo é produto de uma pesquisa de Doutorado realizada entre 2015 e 2019, que analisou o processo de reformulação curricular para a formação de professores do nível primário na província de Buenos Aires. Neste texto, levou-se em consideração a hipótese de que esse processo envolve uma reestruturação dos sentidos do ensino, a partir da centralidade da categoria "práxis" na formação profissional. A análise de fontes primárias, a bibliografia especializada bem como as entrevistas com os sujeitos envolvidos permitiram afirmar que o currículo é entendido como uma prática discursiva que busca configurar identidades, embora seja possível observar a resistência que os professores estabelecem nesse sentido, durante o desenvolvimento curricular na jurisdição entre 2004 e 2007, um processo de negociação permanente entre a interpelação oficial e os destinatários da referida política.

Palavras-chave: Formação de professores. Currículo. Práxis.

\begin{abstract}
This article is a product of Doctoral research carried out between 2015 and 2019, which analyzed the process of curricular reformulation for the training of primary school teachers in the Province of Buenos Aires. In this text, it was taken into consideration the hypothesis that this process implied a restructuring of the meanings of teaching, based on the centrality of the category "praxis" in professional training. The analysis of primary sources, specialized bibliography as well as the interviews carried out with the subjects involved allowed us to affirm that curriculum is understood as a discursive practice that seeks to configure identities, although it is possible to observe the resistance that teachers establish in this respect, during the curricular development in this jurisdiction between 2004 and 2007, a process of permanent negotiation between the official interpellation and the subjects to whom this policy is addressed.
\end{abstract}

Keywords: Teacher training. Curriculum. Praxis.

\title{
Introducción
}

En el marco de una serie de reformulaciones de las políticas educativas, entre las cuales se incluye la Ley de Educación Nacional No 26.633 (2006) a partir del año 2004 la Provincia de Buenos Aires llevó adelante un proceso de consulta a los actores educativos (funcionarios de gobierno, asesores, docentes, estudiantes, directivos e inspectores) respecto de la formación docente, a fin de formular una propuesta curricular superadora de las políticas educativas neoliberales que aún se encontraban en vigencia. El "Proceso de Redefinición Curricular" implicó, en una primera etapa, una gran consulta a la comunidad educativa que se extendió desde los años 2004 a 2007 cuyo resultado fue el nuevo Diseño Curricular para el Profesorado de Educación Primaria (2007a). Es necesario señalar que el proceso continuó durante los primeros años de implementación efectiva de la nueva política curricular en los Institutos Superiores de Formación Docente [ISFD] mediante políticas desarrolladas por la Dirección de Educación Superior de la provincia, con estrategias de "acompañamiento" bajo jornadas de intercambio y talleres, con la utilización de los llamados "Módulos de acompañamiento capacitante".

El Diseño Curricular para el Profesorado de Educación Primaria 2007 [DC] estableció una serie de modificaciones, entre las cuales se destacan:

- La duración de la carrera, que se extiende de tres a cuatro años.

- El campo de práctica docente incluye dos ejes, la "Prácticas en terreno" y las "Herramientas de la práctica", que se configuran como ejes transversales de todos los años, focalizado en diversos espacios sociales, no sólo en el escolar.

- Se genera el Taller de Integración Interdisciplinar (TAIN), como un espacio curricular transversal a todas las materias en cada uno de los años, compuesto por 4 horas reloj mensuales, con el objeto de brindar tratamiento a las diversas problemáticas y/o temáticas que cada institución y/o estudiantes y docentes consideren oportunas tratar en función del eje mencionado para cada año de la carrera.

Práxis Educativa, Ponta Grossa, v. 16, e2116583, p. 1-18, 2021

Disponível em: < https://www.revistas2.uepg.br/index.php/praxiseducativa> 
El diseño curricular está organizado alrededor de cinco Campos y Trayectos opcionales, vinculados por preguntas centrales y organizadores de relaciones entre las diferentes Materias que los componen:

- Campo de Actualización Formativa. ¿Qué aspectos de la formación previa es necesario profundizar para transitar la formación docente?

- Campo de la Fundamentación. ¿Cuál es el sentido de la docencia en el mundo actual en la sociedad latinoamericana y argentina?

- Campo de la Subjetividad y las Culturas. ¿Qué saberes permiten el reconocimiento y la comprensión del mundo subjetivo y cultural del sujeto de la educación?

- Campo de los Saberes a Enseñar. ¿Cuáles son los núcleos de saberes significativos y socialmente productivos que se articulan en la enseñanza?

- Campo de la Práctica Docente. ¿Cuáles son los recorridos formativos necesarios para asumir una praxis transformadora de la práctica docente?

- Trayectos Formativos Opcionales. ¿Cuáles son los recorridos complementarios de la formación que percibe y propone cada Institución? (Diseño Curricular, 2007a, p. 27 y sig.). ${ }^{1}$

La nueva política curricular presenta una extensa fundamentación teórica donde la enseñanza es entendida como proceso cultural, político y pedagógico, así como recupera el sentido latinoamericanista en el campo educativo, constituyéndose a partir de categorías de las teorías pedagógicas críticas en su desarrollo. La categoría "praxis" recorre ampliamente el documento y se convierte en el eje estructurante del mismo, por lo cual resulta factible señalar que existe allí un intento por configurar un discurso educativo que apela a la reflexividad y la transformación de un orden social desigual mediante la educación, entendida ésta como derecho.

Por otro lado, los documentos de discusión utilizados durante el proceso de consulta, así como el Diseño Curricular, se apoyan en nociones posestructuralistas del curriculum, recuperando categorías de Alicia de Alba, Rosa Nidia Buenfil Burgos y Margarita Pansza González, remarcando la importancia de la subjetividad, lo cual nos permite sostener que se producen interpelaciones distintas desde el campo pedagógico y político hacia los docentes en formación. En este último punto, es importante señalar que el Diseño Curricular propuesto, dividido en los Campos mencionados, establece la configuración de un campo específico de saberes a enseñar en relación a las subjetividades, transversal a la carrera. En el documento analizado, la práctica docente se conforma como objeto de transformación, dentro del Campo de la Práctica Docente, en que se articulan los campos restantes, produciéndose una mutua interpelación y transformación entre ellos. Desde esta perspectiva, el documento apela a la constitución de un posicionamiento docente, entendido como el acto político que configura su función. Desde este lugar, asume la relación entre educación y política en función de favorecer el ejercicio de prácticas educativas fundadas en la praxis docente, como análisis crítico, situado, contextuado, considerando las múltiples variables que atraviesan a la educación como proceso históricamente condicionado y determinado.

La tesis doctoral de la cual se desprende este artículo tuvo como objetivo analizar los sentidos que articulan el Diseño Curricular para el Profesorado de Educación Primaria, así como las interpelaciones que realiza a los docentes en formación, sumando la particular recepción que tuvo por parte de los sujetos destinatarios por entender que allí siempre existe una disputa por sus sentidos. (SOUTHWELL, 2013, 2015). En los siguientes apartados nos centraremos particularmente en los sentidos que cobró la educación, comprendida como acto político, la centralidad de la enseñanza y su relación con la noción de praxis, asociada a la de "horizonte

${ }^{1}$ GOBIERNO DE BUENOS AIRES. DIRECCIÓN GENERAL DE CULTURA Y EDUCACIÓN.

Diseño Curricular para la Educación Superior. Niveles Inicial y Primario. Buenos Aires. 2007. En adelante se referenciará (Diseño Curricular, 2007a).

Práxis Educativa, Ponta Grossa, v. 16, e2116583, p. 1-18, 2021

Disponível em: <https://www.revistas2.uepg.br/index.php/praxiseducativa> 
La recuperación de la praxis en el desarrollo curricular de la formación docente. El caso de la província...

formativo" y, finalmente, nos ocuparemos de uno de los interrogantes centrales de la investigación relativo a la posibilidad de prescribir la "praxis" en el sentido freireano del concepto.

\section{Metodología de investigación}

En tanto se procuró realizar un análisis exhaustivo de los sentidos que la política curricular estudiada estableció en cuanto a la formación y el rol docente, se realizó la búsqueda y análisis de la documentación previa a su implementación, así como se entrevistó a asesores, funcionarios de la gestión provincial y directivos y docentes de diversas regiones de la Provincia de Buenos Aires², con el fin de considerar cuáles fueron los debates que se suscitaron durante el proceso de consulta, las ideas que los constituyeron y que procuraron organizar tanto las discusiones previas a la nueva formulación curricular como el propio Diseño Curricular. En este sentido, las entrevistas realizadas a las funcionarias y asesoras curriculares de la gestión provincial son de tipo no estructuradas, con el propósito que las participantes pudieran desarrollar su visión del proceso de redefinición curricular, observando sus complejidades, rupturas y tensiones. Las entrevistas realizadas a los docentes y directivos del Nivel Superior, en cambio, fueron semi estructuradas a fin de obtener información que permitiera realizar un análisis comparativo y denso respecto de algunos aspectos considerados centrales, seleccionando tres regiones educativas ${ }^{3}$ de la Provincia de Buenos Aires, de acuerdo a la diversidad contextual, económica y social de la misma, a fin de obtener una muestra representativa. De este modo, se entrevistó a directivos y docentes de la Región 1, que comprende entre sus distritos a la capital provincial, la Región 4, compuesta por distritos pertenecientes a la zona sur del Gran Buenos Aires, y de la Región 11, comprendida por cinco distritos de la zona noreste de la provincia, compuestos por zonas urbanas y semi-rurales) ${ }^{4}$. En este sentido, las entrevistas se realizaron mediante el consentimiento informado de los participantes estableciendo la garantía de privacidad. (MAINARDES; CARVALHO, 2019).

Por lo tanto, el abordaje metodológico es principalmente cualitativo, ya que se procuró generar conocimiento a partir de los datos empíricos recogidos en el trabajo de campo. Se enfatizó un proceso en espiral de ida y vuelta constante entre obtención de datos y generación de teoría, a través del cual se focalizó en los aspectos más sustantivos del problema de investigación planteado (GLASER, STRAUSS, 1967; SAUTÚ, 2003; SIRVENT, 1995; TAYLOR, BOGDAN, 1986).

La metodología empleada fue el rastreo bibliográfico, selección de textos y análisis del corpus bibliográfico, incluyendo el análisis de los textos primarios de referentes del enfoque teórico empleado. La opción metodológica se concentra en el Análisis Político del Discurso [APD], como perspectiva analítica posfundacional y no-esencialista, retomando los aportes de autores tales como

\footnotetext{
${ }^{2}$ En la República Argentina, la Provincia de Buenos Aires se conforma por 135 partidos, de los cuales 24 corresponden al "Gran Buenos Aires", región metropolitana que rodea a la Ciudad Autónoma de Buenos Aires. Según el censo 2010, desarrollado por el Instituto Nacional de Estadística y Censos [INDEC], es la provincia más densamente poblada del país. Asimismo, en el Gran Buenos Aires se concentra la mayor densidad poblacional. Los 121 partidos correspondientes al interior de la provincia se caracterizan por poseer una densidad mucho menor de habitantes, conformándose en zonas rurales y semi-rurales, aunque cuenta con centros urbanos importantes entre los que se destacan los partidos de la costa atlántica, dedicados mayormente al turismo, así como la capital provincial, La Plata, ubicada a sesenta kilómetros de la Ciudad Autónoma de Buenos Aires.

${ }^{3}$ La Provincia de Buenos Aires se organiza en veinticinco regiones educativas que agrupan a los 135 municipios que la componen. Las regiones educativas son concebidas como la instancia de conducción, planeamiento y administración de la política educativa. Cada Región comprende a uno o más de un distrito conforme a los componentes comunes que los agrupen (Ley de Educación Provincial No 13.688, art. 58. 27 de junio de 2007c)

${ }^{4}$ De los cinco partidos que componen la región 11, dos de ellos forman parte de los 30 partidos que el Instituto Nacional de Estadística y Censos [INDEC] denomina "partidos del aglomerado Gran Buenos Aires" pero no forman parte de los 24 partidos que el INDEC denomina "partidos del Gran Buenos Aires” en sentido administrativo.
}

Práxis Educativa, Ponta Grossa, v. 16, e2116583, p. 1-18, 2021 Disponível em: <https://www.revistas2.uepg.br/index.php/praxiseducativa> 
Ernesto Laclau, Chantal Mouffe, Slavoj Zizeck, Jacques Lacan, Antonio Gramsci, Louis Althusser, referidos a las nociones de "identidad", "hegemonía" e "interpelación" y, particularmente dentro del ámbito educativo, los trabajos de Myriam Southwell, Rosa Nidia Buenfil Burgos, Alicia de Alba, Eloísa Bordoli, William Pinar, Alice Casimiro Lopes, Cleo Cherryholmes, Alejandro Vassiliades, entre otros, intentando vincular dichas nociones en el campo educativo general y, en particular, a la identidad docente. Se procedió al relevamiento y recopilación de fuentes documentales relativas a la política educativa, específicamente los documentos curriculares para la educación superior en la provincia de Buenos Aires, y de las circulares y comunicaciones elaboradas a propósito de dicha implementación. Estas tareas permitieron reconstruir el marco más amplio de la política educativa en la que se inserta la propuesta curricular que actualmente se desarrolla en la provincia.

\section{La educación como acto político: escolarización, praxis y rol docente}

Una noción que atraviesa los lineamientos curriculares prescriptos por el Estado provincial, es la "praxis" como eje estructurante de la práctica docente. Asimismo, se pueden observar referencias en función de favorecer la criticidad de los sujetos en pos de democratizar la educación, en un marco donde ésta es situada como derecho. El nuevo diseño curricular apela al desarrollo docente como trabajador cultural, como productor cultural, sujeto político, con perspectivas crítico-transformativas y alto compromiso con sus comunidades y la educación pública. Para ello, buscó desplegar "horizontes formativos", noción que se estructura bajo tres ejes, tomando en consideración al maestro/a como "profesional de la enseñanza", como "pedagogo" y como "trabajador de la cultura". Así en la concepción de los "horizontes formativos", el Diseño Curricular [DC] postula

[...] - es necesario proponer trayectorias formativas en las que se aporte a dos construcciones:

- la del posicionamiento docente en la comunidad, en el campo y el sistema educativo, en la institución escolar y en el trabajo educativo áulico concreto, situado en sus expresiones singulares, y la de la recuperación del sentido de la docencia en nuestras sociedades en crisis y en contextos de transformaciones culturales continuas;

- si la educación pública, desde la época fundacional del sistema educativo argentino y en los tiempos posteriores de fuerte pregnancia homogeneizadora, fue la forma predominante de política cultural, necesitamos recuperar el papel político-cultural de la educación pública en la actualidad. Para ello, debemos reconocer otros polos desde los cuales se producen políticas culturales (en el mismo sentido, o no, que el de la educación pública) y proponer unas trayectorias formativas en las que el docente incorpore de modo crítico y reflexivo saberes y actitudes que le permitan actuar educativamente, y con sentido político-cultural, en los nuevos contextos;

- en su formación básica, el docente tiene no sólo que conocer lo que va a enseñar y cómo enseñarlo, sino reconocer a quién se lo va a enseñar, teniendo en cuenta las propias características subjetivas y los contextos y polos socioculturales y ambientales en los cuales ese sujeto se constituye y se forma, reconociendo a su vez el carácter ético-político y sociocultural de su profesión;

- un docente en formación tiene que habilitarse para:

- "leer" la experiencia y el mundo más allá de las miradas escolares tradicionales;

- formarse para comprender su situación profesional y construir conocimientos en el contexto de un grupo;

- experimentar un nuevo modo de trabajo colectivo y en colaboración

- el diseño propicia, desde el espacio de integración y construcción interdisciplinaria de cada año, la profundización - la articulación práctica - teoría (praxis) (Diseño Curricular, 2007a, p. 18-19).

Nos detendremos en estos conceptos a partir de los planteos de Paulo Freire.

Práxis Educativa, Ponta Grossa, v. 16, e2116583, p. 1-18, 2021

Disponível em: <https://www.revistas2.uepg.br/index.php/praxiseducativa> 
La recuperación de la praxis en el desarrollo curricular de la formación docente. El caso de la província...

La noción "praxis educativa" no resulta una novedad las políticas curriculares en Argentina, dado que fue ampliamente divulgada en la formación docente promovida por la reforma educativa en Argentina en la década de 1990. No obstante, en dicho contexto histórico, fue presentada desde una simplicidad que soslayaba el sentido político de la concepción teórica formulada por su autor. Ya en "Pedagogía del oprimido" ([1970] 2005) Freire supone la praxis dentro de su mirada dialéctica de las prácticas pedagógicas que apelan, sustancialmente, a lo que llama proceso de humanización. De este modo, el autor supone que la educación "problematizadora" será la única capaz de superar la dialéctica opresor-oprimido, a fin de lograr la liberación de ambos, percibiendo a la praxis como "La palabra, como comportamiento humano, significante del mundo, no sólo designa a las cosas, las transforma; no es sólo pensamiento es "praxis"” (FIORI, 2005, p. 24). Al comprender al hombre como "ser inconcluso", el autor supone que la deshumanización presente en el mundo "[...] es distorsión de la vocación de SER MÁS" (FREIRE, 2005, p. 40), distorsión que conduce a los oprimidos a luchar contra los opresores. Ahora bien, esta lucha sólo puede darse con objeto de la liberación de la conciencia opresiva presente en los oprimidos, en tanto "[...] restauradores de la humanidad de ambos" (Ídem, p. 41). Asimismo, señala el miedo a la libertad tanto en los oprimidos como en los opresores planteándolo del siguiente modo: "En los oprimidos el miedo a la libertad es el miedo de asumirla. En los opresores el miedo de perder la 'libertad' de oprimir" (Ídem, p. 44). Cuando el opresor dificulta al oprimido en su "búsqueda de afirmación como persona", se materializa un acto de violencia al herir "[...] la vocación ontológica e histórica de los hombres: la de ser más" (Ídem, p. 56). Por lo cual, el autor postula que "[...] lo importante es que la lucha de los oprimidos se haga para superar la contradicción en que se encuentran; que esta superación sea el surgimiento del hombre nuevo, no ya opresor, no ya oprimido sino hombre liberándose" (Ídem, p. 57). Allí supone que la acción pedagógica debe situarse en función de la solidaridad con los oprimidos, pero asumiendo que dicha solidaridad no debe implicar una situación paternalista, dado que ello conduciría a reproducir las prácticas pedagógicas de la "educación bancaria", cuya consecuencia única solo puede ser mantener a los oprimidos en la misma situación de dependencia.

En el Diseño Curricular analizado, si bien la "praxis docente" estructura el Campo de la Práctica en cada uno de los cuatro años del profesorado, esta noción es particularmente trabajada a partir de la fundamentación de la asignatura "Campo de la Práctica 1", correspondiente al primer año del mismo. De acuerdo con ello, se comprende:

En la Práctica en terreno de 1er. Año, se realiza una experiencia social, en la que el futuro docente se vincule con el campo sociocultural de la comunidad, a través de las organizaciones de la misma, en una práctica educativa no escolarizada y no reducida a acciones de apoyo escolar. Tiene por objeto acceder a la práctica docente desde la comprensión y el posicionamiento del futuro docente en el campo educativo. Esta estrategia alienta un vínculo de colaboración orgánica que favorezca la contribución social, cultural y educativa para el desarrollo local, a la vez que el encuentro sistemático con la sociedad, la cultura y el conocimiento contemporáneos (cf. MECyT, 2005: 12 y 26). Por otra parte, promueve la construcción de una perspectiva que permita repensar la práctica educativa y la propia tarea docente en el marco de las transformaciones culturales, políticas y sociales que se plantean hoy en nuestro país y en el mundo contemporáneo (MECyT, 2005: 28). Finalmente, reconoce otras alternativas de formación en otras tareas y roles docentes, más allá del ámbito del aula o de la gestión y conducción escolar (cf. MECyT: 2005: 29). Desde este enfoque, se asume una doble evidencia: que lo educativo no pasa hoy sólo por las instituciones escolares; y que el educador debería comprender la relación teoría-praxis no sólo como 'educativa' en un sentido restringido, sino como ampliada a lo 'educativo-social' (Diseño Curricular, 2007a, p. 57).

Al incorporar estos sentidos de transformación social, el Diseño Curricular sostiene una conceptualización de la educación que recupera explícitamente la concepción freireana. En efecto, la referencia al autor se evidencia en gran cantidad de conceptos y significantes que recorren la

Práxis Educativa, Ponta Grossa, v. 16, e2116583, p. 1-18, 2021

Disponível em: <https://www.revistas2.uepg.br/index.php/praxiseducativa> 
propuesta pedagógica. De este modo, no sólo la noción de praxis es el eje de la práctica docente, sino que la definición de los "horizontes formativos" bajo nociones distintas pero relacionadas entre sí apoyan dicha perspectiva. Así, el Diseño Curricular supone que el docente se configura bajo tres dimensiones: el "maestro como profesional de la enseñanza", "el maestro como pedagogo" y "el maestro trabajador de la cultura" (Diseño Curricular, 2007a, pp. 16-17). En este sentido, puede leerse lo prescripto en relación con los planteos de Henry Giroux (1990) respecto de las interpelaciones realizadas por las reformas educativas hacia los docentes. Desde este lugar el autor supone que, en líneas generales, los profesores han sido interpelados como sujetos cuyas actividades principales se asocian a la burocracia escolar, como "[...] técnicos superiores encargados de llevar a cabo dictámenes y objetivos decididos por expertos totalmente ajenos a las realidades cotidianas de la vida del aula." (Ídem, p. 171). De este modo, plantea la categoría de "intelectual transformativo" bajo tres argumentos. El primero de ellos relacionado a modificar la interpelación realizada desde los expertos dentro de las pedagogías de la gestión o tecnocráticas, en favor de realizar un examen del trabajo de los docentes como una tarea intelectual. En segundo término, invita a pensar sobre las condiciones ideológicas y materiales que resultan necesarias para pensar a los profesores como intelectuales. Por último, según Giroux, esta noción "[...] contribuye a aclarar el papel que desempeñan los profesores en la producción y legitimación de diversos intereses políticos, económicos y sociales a través de las pedagogías que ellos mismos aprueban y utilizan" (Íbidem, p. 176). Pensar a los profesores como intelectuales transformativos implica mucho más allá que la reflexión crítica sobre la propia práctica. Supone contemplar la educación en general como un acto político, que favorezca una posibilidad real de transformación social hacia una sociedad democrática y un orden social más justo, así como denunciar las formas de dominación, a la vez de tomar conciencia de las mismas, de modo de poder pensar y llevar a cabo acciones transformadoras. Esta noción puede encontrarse en la idea del "maestro como pedagogo" que es, precisamente, uno de los ejes de los "horizontes formativos"

Como pedagogo, el maestro/a se encuentra hoy atravesado por la antigua dicotomía entre tekné (el hacer calculable y ciertamente ordenado) y poiesis (la creación estética y la producción de un mundo o un orden posible, distinto al existente). Dicho de otro modo, ha ganado terreno la idea del maestro/a regido por una racionalidad pedagógica eminentemente instrumental (calculadora, ordenada, dirigida por la previsibilidad). Se trata de incorporar, como horizonte formativo, la racionalidad pedagógica comunicativa (para seguir las ideas de J. Habermas) centrada en la intersubjetividad y la problematización. Un tipo de racionalidad que no deja en manos únicamente de los especialistas en educación la producción y la reproducción del discurso pedagógico, sino que asume el desafío de articular con su acción la reflexión enriqueciendo el campo de la reflexión teórica en su propia práctica, a través de la problematización que realiza en comunicación con otros. Retomando luego su propia práctica, la teoría y la práctica educativas, en una suerte de espiral dialéctica permanente. Por otra parte, la complejidad actual asedia los modelos pedagógicos demasiado "racionales" o abstractos. Pero "la complejidad es el desafío, no la respuesta" como dice E. Morín. Por ello, solo una pedagogía basada en el diálogo de saberes, el pensamiento crítico y la incerteza pueden viabilizar la re-unión entre tekné y poiesis, desinstrumentalizando la racionalidad pedagógica y dotándola de nuevos instrumentos. (Diseño Curricular, 2007a, p. 17).

De igual modo, entender al docente como "profesional de la enseñanza" supone, en primer lugar, una concepción particular de la misma, como proceso con características profundamente distintas a la "educación bancaria" (FREIRE, 2005) que implica la centralidad de la misma en la tarea, anclada en un contexto social-cultural más amplio. De acuerdo con ello, la propuesta curricular entiende que "el propósito político formativo de fortalecer la identidad, la presencia y la significación social de la profesión docente implica desarrollar un análisis histórico-crítico de la práctica profesional docente (atendiendo a las necesidades sociales, culturales, políticas, educativas y del mundo laboral-profesional)" (Diseño Curricular, 2007a, p. 16). Asimismo, el Diseño

Práxis Educativa, Ponta Grossa, v. 16, e2116583, p. 1-18, 2021

Disponível em: < https://www.revistas2.uepg.br/index.php/praxiseducativa> 
La recuperación de la praxis en el desarrollo curricular de la formación docente. El caso de la província...

Curricular reconoce que esta dimensión de la tarea docente implica las tensiones con las cuales se configura dicha dimensión, explicitando:

Como enseñante el maestro/a se encuentra experimentando una tensión entre:

- la idea dominante de "enseñanza" como transmisión y la noción de enseñanza como proceso de reconstrucción cultural y de la propia transmisión. En este sentido, la transmisión es un contacto con una herencia cultural, no ya un acto mecánico de control disciplinador, sino una experiencia de un acto en común de habilitación del otro para resignificar lo recibido;

- la idea dominante de la "enseñanza" referenciada en el docente y la noción de múltiples espacios, circunstancias y sujetos referenciales que influyen en el proceso de aprendizaje, por lo cual no existe linealidad entre ambos procesos;

- la idea dominante de la "enseñanza" ligada al rol individual del docente en la cultura escolar y la noción de la enseñanza como un proceso compartido y social (cf. Coscarelli, 2007). (Diseño Curricular, 2007a, p. 16).

Por otro lado, los sentidos otorgados en torno a la noción de "intelectual transformativo" (GIROUX, 1990) también se observan en otra de las dimensiones mencionadas por la política curricular en la configuración del rol: la comprensión del "docente como pedagogo", que, distanciándose de las nociones tecnocráticas, recupera la perspectiva comunicativa habermasiana, entendiendo, asimismo, el pensamiento crítico y complejo postulado por Edgar Morin.

En relación a la última dimensión propuesta por la política curricular, el docente como "trabajador de la cultura" supone la recuperación del carácter político-cultural de la institución educativa y, desde esa comprensión, el docente es "[...] un actor clave para reconstruir el sentido sociopolítico de la escuela pública y de la educación pública como una política cultural inclusiva [...] (Diseño Curricular, 2007a, p. 17). De este modo, el rol docente como trabajador cultural implica un rol activo de los maestros y las maestras y una consideración de las perspectivas interculturales.

La consideración de la amplitud, la complejidad y el sentido de la práctica docente incluye un posicionamiento social y el reconocimiento de la dimensión política de la docencia. En este sentido es que consideramos al maestro/a como trabajador cultural, como transmisor, movilizador y creador de la cultura. Las ideas tradicionales han presentado a la cultura como una 'cosa' frecuentemente acabada y como un elemento/insumo factible de ser enseñado. Sin embargo, más que nunca resulta necesario repensar en la actualidad el carácter político-cultural de la escuela y el rol del docente como trabajador de la cultura. Para ello es necesario que el maestro/a pueda reconocer e interactuar con otros espacios y otros trabajadores de la cultura, atendiendo a que la cultura no es algo cosificado o muerto, sino que está en constante dinamismo y recreación. No es 'la' cultura sino 'las' culturas. De igual modo, es imprescindible hacerlo desde una percepción crítica de las tensiones entre diversidad y hegemonía cultural. (Diseño Curricular, 2007a, p. 17).

Como señalábamos al comienzo, la centralidad de la enseñanza no remite al proceso estricto de transmisión, sino supone generar estrategias de transformación social, donde el respeto por la diversidad y el derecho a la educación se encuentre garantizado por el Estado. Encontramos aquí que el significante "inclusión" (SOUTHWELL; VASSILIADES, 2016) aglutinó diversas demandas que se encontraban asociadas a los reclamos de los sindicatos docentes, de la sociedad civil y por supuesto, de los educadores y estudiantes, involucrados desde el comienzo del proceso de redefinición curricular para la formación docente como voces legítimas.

En este sentido, asumimos que la política curricular retoma los planteos realizados por Henry Giroux y Peter McLaren (1998) quienes referencian la necesidad de redefinir la comprensión actual de la relación escuela / sociedad, desde una perspectiva que vaya más allá de los enfoques críticos cuyo análisis sólo se sostienen desde la visión de las escuelas como herramientas de reproducción de la ideología dominante en el marco del capitalismo. Los autores sostienen que “[...] al minimizar la importancia del lenguaje, la experiencia y el poder, los educadores críticos han

Práxis Educativa, Ponta Grossa, v. 16, e2116583, p. 1-18, 2021

Disponível em: <https://www.revistas2.uepg.br/index.php/praxiseducativa> 
abandonado el desarrollo de un discurso que enlace la subjetividad, la pedagogía y la historia con un lenguaje de visión y vida pública" (1998, p.138). Desde la perspectiva que supone que no es posible comprender las escuelas como simples ámbitos de reproducción, Giroux y McLaren plantean que un proyecto de transformación implica la disputa por el vocabulario teórico y ético empleado en el análisis de las instituciones educativas, es decir, un lenguaje crítico de la escolarización donde la ética pueda ser fortalecida, en la cual el poder puede ser utilizado en la "liberación humana" (Íbidem, p. 144). Asumiendo al lenguaje y al conocimiento no sólo como meros descriptores sino como constructores de la realidad, los autores señalan que las formas de escolarización, y por tanto también lo social, son simbólicamente construidas siempre en relación con el ejercicio del poder, suponiendo, asimismo, que "[...] las cosas son signos de las palabras, por lo tanto, no existe realidad social que no sea experiencia a través de la matriz social del discurso" (Ídem, p. 145). De acuerdo con ello, la actual política curricular entiende que reconocer el carácter social e intercultural de cualquier práctica educativa implica " [...] una innovación/transformación pedagógica y curricular que parta [...] no sólo de distintos contenidos o experiencias culturales, sino también de procesos de interacción social en la construcción de conocimientos (Walsh, 2001)." (Diseño Curricular, 2007a, p. 20). De modo concomitante, lo anterior supone "[...] promover relaciones dialógicas e igualitarias entre personas y grupos que participan de universos culturales diferentes, trabajando los conflictos inherentes a esta realidad" (Ídem).

Esta postura puede claramente relacionarse con lo expuesto por Michael Apple (1996) en relación a su análisis de la "política cultural". Para el autor, la política cultural en educación no sólo refiere a cuál es la selección del capital cultural que es considerado socialmente válido y, en tanto tal, se configura en el "saber oficial" sino que también debe referir a las formas que se utilizan como recursos de oposición a las formas y políticas hegemónicas, o bien para implementar nuevas formas que deben plasmarse en las instituciones y en la vida cotidiana. (APPLE, 1996, p. 45-46).

Mediante los planteos anteriores podemos observar en el Diseño Curricular bonaerense una férrea defensa de la idea de la educación como un acto de comunicación mediante el cual se permitiría el desarrollo de la conciencia crítica en el sentido freireano del concepto. Para Freire (2004), la acción pedagógica debe tomar en cuenta la lectura del mundo que tienen los oprimidos, que siempre precede a la lectura de la palabra. Esto no implica imponer el saber del educador como el verdadero sino, muy por el contrario, una tarea fundamental del educador progresista debe ser sensible a la lectura y a la relectura del grupo, provocar a éste y estimular la generalización de la nueva forma de comprensión del contexto, todo ello mediante el diálogo. No obstante, la capacidad de diálogo no niega la validez de momentos explicativos, en que el profesor expone o habla del objeto. Lo fundamental es que profesor y alumnos conozcan que la postura que adoptan es dialógica, abierta, curiosa, indagadora y no pasiva. Lo realmente importante es que educadores y educandos se asuman como seres epistemológicamente curiosos y a este respecto, uno de los saberes fundamentales para la práctica educativa crítica es la necesaria promoción de la curiosidad espontánea a la epistemológica, mediante la "rigorización" de la primera. Por lo cual, para Freire, la educación, entendida de acuerdo a las mencionadas características, es necesariamente inherente a la transformación de las situaciones de desigualdad. Así, supone que la nueva pedagogía debe buscar la toma de conciencia de los oprimidos de su propia situación de opresión, comprometiéndose en la "praxis" con su transformación, mediante la cual resultará indispensable la acción profunda a través de la cual se enfrentará la cultura de la dominación (FREIRE, 2005).

De acuerdo con ello, el planteo acerca de la práctica docente cuyo significante es la "praxis", articula, dentro de la política curricular analizada, otros sentidos que conforman la identificación docente, en tanto trabajador cultural, promotor de la transformación, pero esencialmente, como sujeto con capacidad crítica.

Práxis Educativa, Ponta Grossa, v. 16, e2116583, p. 1-18, 2021

Disponível em: < https://www.revistas2.uepg.br/index.php/praxiseducativa> 
La recuperación de la praxis en el desarrollo curricular de la formación docente. El caso de la província...

Considerar la práctica docente como un objeto de transformación requiere un continuo y difícil proceso de autosocioanálisis, donde se pase de la fluctuación a la articulación entre la práctica docente como objeto y la subjetivación de la práctica docente. Pareciera que la única manera de pensar la práctica docente como objeto de transformación, fuera hacerlo poniendo énfasis en el sujeto de transformación, que compromete, que implica en ello la identidad docente, y que a la vez lo hace en una suerte de desimplicación del sujeto docente de la trama que lo somete, que lo controla, que lo disciplina. Es decir: pensar y trabajar la práctica docente en el marco de la dialéctica sujeción (o sujetación) /subjetivación. Subjetivación para la cual es necesario experimentar un proceso de desnaturalización de la práctica docente. Este proceso de trabajo y pensamiento se produce a partir de la reflexión compleja entre los formadores y con los estudiantes docentes en formación. (Diseño Curricular, 2007a, p. 27)

Como puede observarse el rol docente también supone un rol político de suma importancia. Sin embargo, esto sólo puede materializarse en un contexto más general donde la propias nociones de educación, escolarización y formación postulen un sentido pedagógico que apele a la constitución de sujetos críticos, entendiendo por ello a todos los actores dentro del amplio espectro de la educación formal, es decir, profesores, estudiantes, directores, expertos y funcionarios políticos, lo cual intentaremos desarrollar en el apartado siguiente, bajo la comprensión del curriculum desde una perspectiva política. En síntesis, tal como plantea Rosa Buenfil Burgos (1991):

El sujeto de educación se conforma en la práctica como un sujeto activo y condicionado tanto por las relaciones políticas, académicas, administrativas, jurídicas, etc., que rigen y se debaten en la institución escolar, como por discursos en otros espacios sociales, en la vida cotidiana. En esta medida aparece marcado por diversos antagonismos políticos irreductibles al antagonismo de clase, por lo que cabe señalar la exigencia de comenzar a reflexionar respecto de la multiplicidad de polos de identidades que se imbrican en este proceso. (p. 196).

De acuerdo con ello, es factible reconocer, dentro de la política curricular bonaerense nociones en relación a la educación como productora de identidades, como un proceso social, cultural y político donde confluyen diversos elementos considerados en el mismo nivel de importancia. El Marco General de la Política Curricular (MGPC) de la provincia establece una definición de los "sujetos pedagógicos", entendiéndolos como "sujetos sociales complejos". De este modo, la política curricular supone:

El término sujeto contiene en sí mismo la noción de relación, de estar unido a -sujeto auna psiquis, un lenguaje, una sociedad, una cultura, una historia, un ambiente y a otros sujetos. Sujeción que no ha de entenderse como determinación, sino como condición de identidad. (Marco General de la Política Curricular, 2007b, p. 21). ${ }^{5}$

Dentro de esta cosmovisión, los sujetos pedagógicos se conforman como "sujetos históricos", "sujetos de derecho", así como la explicitación de dicha complejidad es entendida a partir de las relaciones que establecen los sujetos en un contexto social más amplio y diverso. En este sentido, el Diseño Curricular caracteriza a los sujetos pedagógicos analizando la diversidad cultural, familiar, sexual -recuperando la perspectiva de género-, sus relaciones con el trabajo -las cuales no remiten únicamente al "empleo"- y el medio ambiente y la influencia de las nuevas tecnologías de la información y la comunicación (Diseño Curricular, 2007a, pp. 22 y siguientes).

Planteado de este modo, en la concepción del curriculum, la política educativa de la provincia supone una "concepción relacional del sujeto pedagógico" en tanto es comprendida

${ }^{5}$ GOBIERNO DE BUENOS AIRES. DIRECCIÓN GENERAL DE CULTURA Y EDUCACIÓN. Marco General de la Política Curricular. Buenos Aires. 2007. En adelante, se referenciará (Marco General de la Política Curricular, 2007b).

Práxis Educativa, Ponta Grossa, v. 16, e2116583, p. 1-18, 2021

Disponível em: <https://www.revistas2.uepg.br/index.php/praxiseducativa> 
como "[...] la relación que se produce entre diversos sujetos sociales que ingresan a la escuela constituyéndose en ella como educadores/as y educandos- mediada por el curriculum." (Diseño Curricular, 2007a, p. 18). De acuerdo con ello, el curriculum es el que ofrece a dicha relación "[...] su especificidad pedagógica y el que da lugar a los procesos de enseñanza y de aprendizaje” (Ídem) entendidos éstos como procesos diferentes pero complementarios, lo cual nos introduce al tercer eje que configura la política curricular, comprendida como un proceso de disputa de sentidos y, en tanto tal, como un proceso político.

\section{La prescripción de la praxis docente}

Uno de los mayores interrogantes que sustentaron la investigación remitía a pensar si resulta posible prescribir la praxis docente en el sentido freireano del término que, como hemos señalado, se presenta como eje estructurante del Diseño Curricular, comprendiendo el mismo, en tanto documento que "prescribe" las formas en las cuales se debería actuar. En este sentido, el análisis del proceso de Reformulación Curricular que hemos descripto, así como la particular configuración de los "horizontes formativos" que presenta la política curricular han otorgado algunas primeras respuestas. Sin embargo, creemos necesario recuperar las respuestas que nos concedieron los diversos actores involucrados en el proceso, por considerar que allí se pueden evidenciar las disputas que se produjeron en la interpretación que cada sujeto, desde su rol particular, le otorgó a dicha categoría.

Retomando la distinción entre normatividad y prescripción realizada por Sofía Picco (2014), quien señala que la primera noción alude y comprende aspectos valorativos en tanto la prescripción se orienta hacia la consecución de los fines propuestos de la manera más eficiente, y se asocia a la expansión de las teorías tecnicistas en educación, asumimos que la política curricular en análisis se inscribe en la "normatividad". En este sentido, al consultarle a la asesora curricular sobre la posibilidad de cierta autonomía de los docentes al ejercer la praxis, la entrevistada se orienta hacia dicha distinción entre normatividad y prescripción del siguiente modo:

[...] hay dos maneras de entender tu pregunta: si uno puede prescribir en el sentido de prescripción cerrada, yo creo que es contradictorio a una mirada crítica y poscrítica de lo que debe ser un curriculum. Ahora desde el punto de vista normativo sí, porque todo curriculum prescripto es una formalidad que orienta, no en un sentido estrecho, sino en el sentido que aún como debe ser la orientación en pedagogía y en didáctica. Es una orientación no rígida, pero orientación al fin [...] esta idea de horizonte formativo es coherente; en otras instancias también habla de la apertura, que cada TAIN (Talleres de Integración Interdisciplinar) organice su propio proyecto. (Entrevista a Asesora Curricular)

Asimismo, la Directora Provincial de Educación Superior y Capacitación Educativa, señalaba que el tema había generado muchas tensiones, sobre todo, en el Campo de la Práctica Docente 1, donde los estudiantes realizan sus prácticas profesionales por fuera del sistema educativo formal, noción concordante con la categoría "praxis" de Paulo Freire, y que, a su vez, puede asociarse a otro de los ejes de los "horizontes formativos" señalados por el Diseño Curricular: el docente "como trabajador de la cultura". Al respecto, la entrevistada también señala cuáles fueron los debates que se suscitaron al pensar la prescripción y la normatividad, pero asociadas a la articulación particular que dichas nociones contienen en la distinción de la idea de "perfil o rol" y la de "horizonte formativo".

[...] Cuando iniciamos esta discusión sobre los horizontes formativos la primera cuestión que apareció era la de definir qué es un horizonte formativo y por qué nos desplazábamos del concepto de perfil y de rol. Lo cual no significa que no haya orientaciones. Lo que trata de recoger es el movimiento tendencial histórico de un proceso en construcción

Práxis Educativa, Ponta Grossa, v. 16, e2116583, p. 1-18, 2021

Disponível em: <https://www.revistas2.uepg.br/index.php/praxiseducativa> 
La recuperación de la praxis en el desarrollo curricular de la formación docente. El caso de la província...

donde hay un horizonte posible pero donde hay en el camino, un movimiento de divergencia, de posibilidad, de apertura en el que no hacés una programación de sujetos [...] estás construyendo subjetividades, posicionamientos, discursos, representaciones y prácticas dotadas de una capacidad de problematizar, de desnaturalizar, de resignificar que además no es algo terminado de una vez y para siempre, sino que tiene esta capacidad permanente de ir modificándose. De la mano de eso la pregunta es: ¿cuáles son los horizontes formativos de la docencia? ¿Qué estudiantes en formación -la misma noción docentes en formación, implica que ya sos un docente- y qué perfiles formativos para ese docente? y luego ¿qué asignaturas, qué comprensiones del campo, qué experiencias hay que atravesar, qué lecturas, qué prácticas para poder construir esos horizontes? [...] Ahí existió una concepción histórica que se configuró en torno a la idea del docente como trabajador. Ese sí es un posicionamiento político muy contundente del campo educativo, que es pensar al docente en su dimensión de trabajador [...] ¿qué tipo de trabajador?, un trabajador del campo de la cultura, un trabajador del campo de la educación. Ahí también hay un desplazamiento del concepto del sistema educativo a campo educativo, que es lo que permite en primer año que la materia ocurra sobre el campo educativo y no solo sobre el sistema. Después se va ingresando al sistema, pero no desde una mirada didáctica sino desde una mirada micropolítica de la escuela [...] La posibilidad de politizar tiene que ver con eso, entender que con subjetividad se escribe la historia y cuáles son los determinantes de la historia. Hay un déficit de un lado y del otro, uno es cultural y el otro es específicamente técnico didáctico, pero los dos enlazan las puntas de lo mismo de un lado y del otro. [...] La de productor de discurso pedagógico, la de productor de conocimiento es una dimensión, un horizonte controversial porque para que esto sea posible, esa producción individual y colectiva de saber. ¿Qué saberes produce un docente? [...]-ahí hay un espacio en el que cuando hablamos del docente como productor hablamos en términos de que uno, colectiva e individualmente, a través de lo que hace, genera experiencia y saberes. [...] lo que se pretende interrumpir es la idea de que el saber siempre está afuera, que es muy propio de las tradiciones dominantes en el campo académico. (Entrevista a la Directora Provincial de Educación Superior y Capacitación Educativa)

Retomaremos un aspecto en particular señalado por la entrevistada respecto de las resistencias que se produjeron sobre todo al introducir la noción de praxis, asociada a la educación como práctica cultural, en el Campo de la Práctica 1 de la carrera. De este modo, en las entrevistas realizadas a directoras y docentes pudimos observar posturas dispares. En algunos casos, la idea de "salir del ámbito escolar" se visualizó como la posibilidad de ampliar el horizonte de posibilidades de un docente en formación, en el sentido de ampliar sus conocimientos, pero, ante todo, ampliar "su mirada", su interpretación, su subjetividad al momento de ingresar a la institución escolar. Por el contrario, en otros casos, las respuestas remitían al obstáculo que ello generaba para la propia "formación docente", considerando que de acuerdo a lo estipulado en el Diseño Curricular los estudiantes ingresan "al aula" recién en el tercer año de la carrera, lo cual dificulta una formación experimentada en dicho ámbito, que es, en síntesis, el ámbito de su accionar profesional. A continuación, las directoras y docentes entrevistadas señalaban al respecto:

Directora ISFD Región 1: Creo que sobre los ejes no hubo discusión. El área que tuvo más convocatoria fue el área de Práctica, que fue muy discutida, había un fuerte posicionamiento de profesores de este instituto de pensar la inserción en la propia institución educativa desde un primer año. Básicamente esta era la cuestión en discusión, en este eje centralizado en la práctica. [...] lo que se tradujo en esta institución es que los chicos recién se posicionan en el aula desde el tercer año. Esto es un problema, porque es tardío en una carrera de cuatro años. La opción a lo mejor era hacerlo antes, de hecho, en segundo año fuera de libreto están haciendo intervenciones áulicas, a pesar de que el eje en segundo año es la institución. Porque en realidad el tema es desde dónde se ve la institución y cómo puede mirar un alumno la institución [...] para los alumnos es una cuestión con mucha dificultad, que ya la veníamos observando en el plan anterior porque es una dificultad que no solo no se superó sino que quizás se agravó o profundizó.

Práxis Educativa, Ponta Grossa, v. 16, e2116583, p. 1-18, 2021

Disponível em: <https://www.revistas2.uepg.br/index.php/praxiseducativa> 
Docente Campo de la Práctica Región 11: [...] hay algo que resulta absolutamente novedoso y es que no se plantea sólo en el cambio curricular en términos de curriculum, de la prescripción del plan de estudios, sino que desde el comienzo es un proceso de acompañamiento a la implementación curricular. Fui parte de eso, se inicia con el primer año y a medida que avanzan los años se va acompañando [...] había un trabajo muy comprometido de encuentros con las personas, con equipos, con juntar gente de distintas regiones y ponerse a trabajar en las representaciones, en lo que eso venía a cuestionar de varias situaciones. Lo más novedoso en este Diseño es que la práctica docente como eje vertebrador de la formación es algo que ya viene de los documentos de Nación e incluso de la gestión de los 90, que venía de la mano del BID [...] La diferencia que acá lo que se planteaba era una idea de práctica docente que no era solo lo estrictamente escolarizado, entonces se planteó la gran novedad que era que el primer año de práctica era por fuera del sistema educativo y trabajar cuestiones desde Freire [...] cuando decía que todo el equipo de la $\mathrm{DES}^{6}$ había "puesto el cuerpo" me refiero a que en lugar de ser prescriptivo resultó en ese acompañamiento. [...] que era evaluado de diversos modos. Yo tenía afinidad con estas cuestiones, pero había gente que no. Había una cuestión de trabajar estas resistencias, de generar una cuestión de lógica y que se podían poner a discutir sentidos, lo que se buscaba era generar algún tipo de espacio de ruptura. [...] se pudo ir trabajando esta cuestión de praxis porque ellos (el equipo de gestión) ${ }^{7}$ generaron una praxis en la propia instancia. No hablaban de capacitación sino de acompañamiento capacitante, $[. .$.$] era empezar a hacer algo, volver y poner en discusión cómo había ido y$ cómo se priorizaba eso. Desde ahí hay algo que se vivió, realmente se puso en acto lo que se estaba proponiendo.

Directivo de Región 4: El Campo de la Práctica 1 es un campo para el que no veníamos formados; cuando hay un cambio de diseño curricular no todos los profesores entienden que eso es un desafío y que hay que ponerse a estudiar. [...] me parece que en el momento de la implementación faltó acompañamiento [...] Es lamentable porque el profesor de primer año estaba acostumbrado a la institución escolar [...] En el caso particular de este Instituto, se buscó un recurso humano que supiera manejar esas cuestiones, nos costó, pero llegamos [...] que la práctica sea el eje cultural de la carrera para nosotros no fue una novedad porque teníamos experiencias previas bastantes interesantes [...] el problema que tenemos es que no siempre los alumnos cuando van a las escuelas a observar y a practicar encuentran una coincidencia entre la teoría que les enseñamos y la práctica docente. Nosotros tenemos que predicar una educación problematizadora, dialogante, pero cuando las estudiantes van a practicar, la maestra orientadora no siempre responde a las prescripciones freireanas entonces las estudiantes tienen que acomodarse. También en su vida profesional está demostrado que un poco se institucionalizan.

Consideramos que las experiencias narradas aportan valiosas concepciones, principalmente, sobre dos dimensiones; por un lado, la lectura que se realiza del "acompañamiento capacitante" y, en segundo lugar, el poder de las tradiciones docentes (SOUTHWELL, 1997, 2012). En relación a la primera dimensión, las distinciones parecen centrarse en el abordaje que se produjo de los módulos de acompañamiento. De este modo, en algunos casos, el mismo es ponderado no sólo por su implementación desde la efectiva recepción de los documentos a las instituciones sino por el hecho "de poner el cuerpo por parte del equipo la Dirección Provincial de Educación Superior" -Docente del Campo de la Práctica de la Región 11-, lo cual contrasta con otra de las respuestas, donde si bien se valoriza el hecho de la materialización de medidas de implementación de las

\footnotetext{
${ }^{6}$ Dirección Provincial de Educación Superior de la provincia de Buenos Aires.

${ }^{7}$ Refiere al Equipo de Gestión Educativa Provincial.

${ }^{8}$ En relación a dicho acompañamiento es importante destacar que todos los Institutos Superiores de Formación Docente de la provincia recibieron lo que se llamó "Módulos de acompañamiento" durante la implementación del Diseño Curricular, los cuales se caracterizaban por presentarse con una serie de actividades que apelaban al desarrollo de la problematización de las concepciones pedagógicas en los/as docentes. Particularmente el Módulo para la implementación del Campo de la práctica 1, que resultó ser el más controvertido, puede consultarse en http://www.adeepra.com.ar/documentos/doc_provincia/MODULO.pdf
}

Práxis Educativa, Ponta Grossa, v. 16, e2116583, p. 1-18, 2021

Disponível em: <https://www.revistas2.uepg.br/index.php/praxiseducativa> 
La recuperación de la praxis en el desarrollo curricular de la formación docente. El caso de la província...

modificaciones curriculares se alude a un trabajo que resultó difícil, en tanto no se comprendía completamente y sólo de comprendió a partir de la incorporación al equipo docente de una integrante del equipo de la Dirección Provincial de Educación Superior -Directora de la Región 4.

En relación a la segunda dimensión, puede observarse claramente en las entrevistas, las disputas que los docentes realizan a las prescripciones oficiales, pero, explícitamente, con las propias "tradiciones sedimentadas", "hibridaciones" que conforman la docencia (SOUTHWELL,1997, 2012). De acuerdo con ello, en relación a la prescripción oficial se observan también concepciones opuestas en relación a la salida del ámbito del sistema educativo formal durante el primer año de la carrera. Así como señala la funcionaria entrevistada, la "salida del espacio escolar" procuró conformar ciertas subjetividades con una visión de la educación desde una mirada política, intercultural e integradora de las diferencias, en generar "subjetividades", lo cual es muy ponderado por la docente del Campo de la Práctica de la Región 11, quien admite que la transición por espacios diferentes permite a los estudiantes construir otras miradas sobre el espacio escolar, en el que se insertan en los siguientes años de formación. Sin embargo, según las propias entrevistadas, esta situación generó también incomodidades en muchos docentes, en algunos casos, existe un rechazo hacia dicha conformación curricular bajo el fundamento de la "necesidad de los estudiantes de insertarse rápidamente en el aula". Esto puede tener dos lecturas, por un lado, la necesidad específica que los docentes expresan de "conocer con mayor profundidad el espacio escolar -y particularmente, áulico- desde el comienzo de la carrera", pero también lo que ellos consideran la construcción de los saberes y subjetividades en los estudiantes: en el caso de la directora de la Región 4 se menciona cómo los graduados de la carrera a pocos años de recibirse se "institucionalizan" adoptando posturas que podrían orientarse como más "tradicionales" a partir de su propia inserción en los espacios escolares, quien le otorga esta construcción de subjetividad al propio funcionamiento "endógeno" del sistema educativo y al particular rol que cumplen los docentes co-formadores?.

Por lo expuesto, es posible afirmar que siempre que existen movimientos o intentos de reformular la formación docente, los mismos no se generan en un ambiente pacífico, donde efectivamente aquello que se prescribe es acatado. El proceso mismo de redefinición, en tanto intento de redefinición de subjetividades y de identificaciones, provoca diversas reacciones y sólo resulta exitoso, en tanto resulta "bien recepcionados" por los sujetos destinatarios de las interpelaciones realizadas. En síntesis, y en términos de Pilar Padierna (2008), ningún discurso puede abarcar totalmente un espacio social, sino que los sujetos interpelados sólo aceptan los diversos elementos que "llenan" esa falta, es decir, resulta necesario que algún elemento de aquella interpelación ya se encuentre constituida en la propia subjetividad.

\section{Reflexiones finales}

En la investigación de la cual surge el presente artículo, se decidió describir y analizar los sentidos que asumió la nueva política curricular bonaerense, considerando centralmente la recuperación de tres ejes que conformaron de modo general el escenario donde ello resultó posible: la centralidad de la enseñanza, la educación considerada un acto político y la definición y desarrollo curricular desde las corrientes posestructuralistas.

El primero de ellos consistió en una revisión de los nuevos modos de comprensión de la enseñanza, mediante el establecimiento de un distanciamiento de la noción dialéctica "enseñanza-

\footnotetext{
${ }^{9}$ En el ámbito de las prácticas docentes, el docente co-formador es aquel que se encuentra efectivamente a cargo del grupo de estudiantes donde el residente realiza sus prácticas y quien también juega un rol central en tanto habilita o no el desarrollo de determinadas prácticas.
}

Práxis Educativa, Ponta Grossa, v. 16, e2116583, p. 1-18, 2021 Disponível em: <https://www.revistas2.uepg.br/index.php/praxiseducativa> 
aprendizaje", bajo la reformulación de la categoría transmisión a la cual se asoció históricamente dicho término. De este modo, fue factible demostrar que si bien la categoría misma de transmisión no se distancia de la de enseñanza, cobra nuevos sentidos que la configuran como un proceso independiente de la estricta direccionalidad hacia los aprendizajes, (clásica formulación tecnicista/neoliberal) aunque sin por eso perder el horizonte de orientarlo. Por otro lado, ello implicó estudiar, pues, la educación y la enseñanza como actos políticos, emprendiendo el análisis de los aportes teóricos recuperados por la política curricular, en relación a la capacidad de favorecer acciones transformadoras de las desigualdades sociales a partir del hecho educativo. Asimismo, el análisis de los sentidos del proceso de redefinición curricular, nos permite afirmar que la nueva política educativa sostuvo una interpelación distinta realizada a los docentes, quienes fueron interpelados como sujetos sociales ${ }^{10}$ del curriculum, no sólo implicados sino capaces de accionar de modos diversos en dicha transformación. En ese sentido, se analizó específicamente la categoría "praxis docente" desde la concepción otorgada por la Dirección de Educación Superior provincial a la misma y se intentó explorar la particular recepción que tuvo en las instituciones formadoras de docentes, a partir de las entrevistas realizadas a docentes y directoras de las mismas.

El trabajo analítico y de campo permitió, a la vez, dar cuenta de los procesos de reformulación, interpretación y relecturas que los docentes realizan de las políticas curriculares. De acuerdo con lo anterior, es posible postular que la comprensión posestructuralista del curriculum (de Alba, 2013; Lopes, 2019) es la que describe de mejor manera el Diseño Curricular de la Provincia de Buenos Aires para la Formación Docente, entendiendo el mismo como un proceso donde se producen diversas negociaciones entre sujetos, con una configuración abierta e intencionalmente, democrática. En este sentido, así como el discurso respecto de la "crisis educativa" de fines de la década de los años 90 se conformó como el conjunto de demandas que luego se aglutinaron bajo el significante "inclusión" que permitió dar una respuesta a la falla o grieta de la configuración discursiva acerca de la "profesionalización" (SOUTHWELL, 2007, 2008) podemos encontrar la emergencia y la legitimidad otorgada por dicha configuración discursiva hegemónica de ciertas categorías devenidas de las teorías pedagógicas críticas en la conformación de la política curricular provincial.

\section{Referencias}

APPLE, M. Política cultural y educación. Madrid: Morata. 1996.

BUENFIL BURGOS, R. Análisis político del discurso y educación. México: DIECINVESTAV-IPN. 1991.

DE ALBA, A. (coord.) El fantasma de la teoría: articulaciones conceptuales y analíticas para el estudio de la educación. México: Plaza y Valdés.2013

DE ALBA, A. Curriculum: crisis, mito y perspectivas. Buenos Aires: Miño y Dávila. 1995.

FIORI, E. Aprender a decir su palabra. In: FREIRE, P. Pedagogía del oprimido ( $2^{\circ}$ reimp.) México: Siglo XXI Editores. 2005. p. 11-26.

FREIRE, P. Pedagogía de la autonomía. Buenos Aires: Siglo XXI Editores. 2004.

FREIRE, P. Pedagogía del oprimido. México: Siglo XXI Editores. 2005.

\footnotetext{
${ }^{10}$ Recuperamos aquí el sentido que Alicia de Alba (1995) le otorga a dicha categoría.
} 
La recuperación de la praxis en el desarrollo curricular de la formación docente. El caso de la província...

GIROUX, H. Los profesores como intelectuales transformativos. Barcelona: Centro de Publicaciones del M.E.C. y Ediciones Paidós Ibérica. 1990.

GIROUX, H.; MC LAREN, P. Sociedad, Cultura y Educación. Madrid: Miño y Dávila. 1998.

GLASER, B.; STRAUSS, A. The discovery of grounded theory. Chicago: Aldine. 1967.

GOBIERNO DE LA PROVINCIA DE BUENOS AIRES. DIRECCIÓN GENERAL DE CULTURA Y EDUCACIÓN. Educación Superior. Niveles Inicial y Primario. Buenos Aires. 2007 .

Disponible

en:

http://servicios.abc.gov.ar/lainstitucion/organismos/consejogeneral/disenioscurriculares/docu mentosdescarga/dcurricularsuperior.pdf Acceso en: 16 de marzo de 2021.

GOBIERNO DE LA PROVINCIA DE BUENOS AIRES. DIRECCIÓN GENERAL DE CUlTURA Y EDUCACIÓN. Marco General de la Política Curricular. Buenos Aires. 2007b. Disponible en: http://servicios.abc.gov.ar/lainstitucion/organismos/consejogeneral/disenioscurriculares/docu mentosdescarga/marcogeneral.pdf Acceso en: 16 de marzo de 2021.

GOBIERNO DE LA PROVINCIA DE BUENOS AIRES. Ley de Educación Provincial Nº 13.688. 27 de junio de 2007c. Disponible en: http://servicios.abc.gov.ar/docentes/capacitaciondocente/pruebadeseleccion2008/presentacion /Normativa\%20Comun/Ley\%2013688-07.pdf.Acceso en: 16 de marzo de 2021.

GOBIERNO DE LA REPÚBLICA ARGENTINA. INSTITUTO NACIONAL DE ESTADÍSTICA Y CENSOS. Censo Nacional de Población, Hogares y Viviendas. 2010. Recuperado en: https://www.indec.gob.ar/indec/web/Nivel4-Tema-2-41-135._Acceso en: 16 de marzo de 2021.

LACLAU, E.; MOUFFE, C. Hegemonía y estrategia socialista. Buenos Aires: Fondo de Cultura Económica. 2004.

LOPES, A. C. Articulações de demandas educativas (im)possibilitadas pelo antagonismo ao "marxismo cultural". Arquivos Analíticos de Políticas Educativas, Tempe, v. 27, n. 109, p. 1 21, 2019. DOI: https://doi.org/10.14507/epaa.27.4881

MAINARDES, J.; CARVALHO, I. C. M. Autodeclaração de princípios e procedimentos éticos. In: ANPED. Ética e pesquisa em Educação: subsidios. Rio de Janeiro: Associação Nacional de Pós-Graduação e Pesquisa em Educação, 2019. p. 129-132.

PADIERNA, M. P. Interpelación y procesos educativos en movimientos sociales. In: SAUR, D.; DA PORTA, E. Giros Teóricos en las Ciencias Sociales y Humanidades. Córdoba: Comunicarte. 2008. p.107-113.

PICCO, S. Concepciones en torno a la normatividad en la didáctica: un análisis interdisciplinario de obras teóricas didácticas y curriculares en la Argentina, entre 1960 y 1990. 2014. Tesis (Doctorado en Ciencias de la Educación). Universidad Nacional de La Plata, La Plata, 2014.

SAUTÚ, R. Todo es teoría: objetivos y métodos de investigación. Buenos Aires: Lumiere. 2003.

SIRVENT, M. T. El proceso de investigación, las dimensiones de la metodología y la construcción del dato científico. Ficha de la cátedra de Investigación y Estadística Educacional I. Buenos Aires: Facultad de Filosofía y Letras - UBA. 1995

Práxis Educativa, Ponta Grossa, v. 16, e2116583, p. 1-18, 2021 Disponível em: <https://www.revistas2.uepg.br/index.php/praxiseducativa> 
SOUTHWELL, M. Algunas características de la formación docente en la historia educativa reciente. El legado del espiritualismo y el tecnocratismo (1955-1976). In: PUIGGROS, A. (org.) Dictaduras y Utopías en la historia reciente de la educación argentina (1955-1983). Buenos Aires; Galerna. 1997. p. 105-155.

SOUTHWELL, M. Análisis Político del Discurso: posiciones y significaciones para la política educativa. In: TELLO, C. (org.) Epistemologías de la Política Educativa: posicionamientos, perspectivas y enfoques. San Pablo: Mercado de Letras. 2012. p. 349-381.

SOUTHWELL, M. El análisis político del discurso y la cuestión de la politicidad de la educación. In: CONFERENCIA DICTADA EN EL COLOQUIO DE ANÁLISIS POLÍTICO DEL DisCURSO Y EDUCACIÓN, 10,. 2013, Ciudad de México. Anais [...]. Ciudad de Mexico: DIECINVESTAV. 2013.

SOUTHWELL, M. La construcción de posiciones docentes frente a situaciones de desigualdad social y educativa. In: SEMINARIO FIGURAS ACTUALES DE LA SEGREGACIÓN, 1., 2015, Buenos Aires. Anais [...]. Buenos Aires: FLACSO, 2015.

SOUTHWELL, M. Política y educación: ensayos sobre la fijación del significado. In: CRUZ PINEDA, O. y ECHEVARRÍA CANTO, L. (coords.) Investigación educativa. Herramientas teóricas y análisis político del discurso. México: Casa editorial Juan Pablos; PAPDI. 2008. p. $261-296$.

SOUTHWELL, M. Profesionalización docente al término del siglo XX: políticas y nominaciones producidas por organismos internacionales. Historia de la Educación, Buenos Aires, n. 8, p. 261 $-285,2007$.

SOUTHWELL, M.; VASSILIADES, A. Articulaciones y disputas en la trama entre trabajo docente e igualdad educativa: una aproximación histórica. In: BRENNER, G.; GALLI, G. (orgs.). Inclusión y calidad como políticas educativas de Estado o el mérito como opción única de mercado Buenos Aires: Stella. 2016. p. 33-52.

TAYLOR, S.; BOGDAN, R. Introducción a los métodos cualitativos de investigación. Buenos Aires: Paidós. 1986.

WALSH, C. La Interculturalidad en la Educación. Lima, Ministerio de Educación, Programa FORTE-PE, 2001.

Recibido: 07/08/2020

Versión corregida recibida: 17/03/2021

Aceptado: 18/03/2021

Publicado online: 24/03/2021

Práxis Educativa, Ponta Grossa, v. 16, e2116583, p. 1-18, 2021

Disponível em: <https://www.revistas2.uepg.br/index.php/praxiseducativa> 